\title{
Retroactive facilitative effects of ECS on learning an avoidance response in a two-choice situation'
}

\author{
J. H. CASSEDAY \\ INDIANA UNIVERSITY
}

Rats learned to avoid a place where they received grid shock to their feet when the grid shock was followed $10 \mathrm{sec}$. later by electroconvulsive shock. The avoidance learning was significantly greater than for rats that received only grid shock or only electroconvulsive shock.

Electroconvulsive shock (ECS) has been used extensively in animal learning studies in an attempt to clarify the role and time course of memory in learning. As ECS upsets neural processes in some way, rats given ECS following conditioning trials usually fail to learn the response, i.e., "retrograde amnesia." However, much of the evidence for this retrograde amnesia hypothesis has been shown to be equally compatable with an ECS avoidance hypothesis (Coons \& Miller, 1960; Adams \& Lewis, 1962a, b; McGaugh \& Madsen, 1964). Under the assumption that ECS is an aversive stimulus, two conditioning hypotheses have been proposed to account for the fact that rats do learn an avoidance response that is followed by ECS. One notion (Coons \& Miller, 1960) is that ECS may be a fear inducing stimulus, while the other (Adams \& Lewis, 1962a, b) is that the pairing of ECS with the experimental situation results in cue produced competing responses which effectively impair performance of the previously learned response. However the effects of ECS as a possible aversive stimulus have not been fully determined (cf. McGaugh \& Madsen, 1964).

The present experiment was conducted in an attempt to examine the components of ECS, aversive or otherwise, and to test the retrograde amnesia hypothesis in a slightly different situation than heretofore employed. Method

The experiment utilized a 2 by 2 design, in a twochoice situation, in which presence or absence of grid shock in the goal box was varied independently from place of the subsequent ECS treatment, inside or outside the goal box. In addition, there was a fifth comparison group which received grid shock but not ECS.

Fifty-nine male albino rats (100 to 120 days old) were given 24 pretest escape learning trials (four trials per day with an intertrial interval of approximately 1 hr.) from grid shock $(0.9 \mathrm{ma})$ in the start box of a T-maze. Choice of goal arm to which $\mathrm{S}$ escaped was partly forced so that two right and two left turn responses were made on each training day. The T-maze was modified so that it had only a start box and two goal boxes, i.e., a T-maze with runways eliminated. The left goal arm was painted flat black and the right flat white, with the remainder, start box and intersection between start and goal boxes, flat grey. Guillotine doors at the exit of the startbox and at the entrance of the goal boxes were lowered to prevent retracing or to force turns during training when necessary. The grid floor of the start box and intersection was wired for electric current independent of the goal arm grids, allowing current to be turned on (or left off) in the goal boxes at any time after current was applied to the start box. Cut-down alligator clip ECS electrodes were briefly attached to S's ears following the first daily training trial.

Following training Ss were randomly assigned to one of five treatment groups: Group S-grid shock alone; Group EI-ECS alone inside the goal box; Group EO-ECS alone outside the goal box; Group SEI-grid shock followed by ECS inside the goal box; and Group SEO-grid shock followed by ECS outside the goal box. The above treatments were administered only when $S$ entered the "incorrect" goal box. The latter was definedas the box that $S$ entered on the first trial following the last training trial. Thus each $S$ received his respective treatment on this trial and could avoid it on following (testing) trials only if he entered the opposite "correct" goal box. Testing lasted, one trial per day, for 24 trials following the first treatment trials. The grid shock $(0.9 \mathrm{ma}$, for $4 \mathrm{sec}$.) was administered immediately after $S$ entered the goal box. Each $S$ remained in the goal box for $10 \mathrm{sec}$. after cessation of the grid shock. If $\mathrm{S}$ made a correct response, or was in a group not receiving grid shock, he merely remained inside the goal box for $14 \mathrm{sec}$. without receiving grid shock. For the groups receiving ECS (50 ma, for 200 msec.), it was given immediately after the $14 \mathrm{sec}$. in the goal box following an incorrect response. The ECS electrodes were always briefly attached to all Ss in each group regardless of whether ECS was to be administered or not.

\section{Results and Discussion ${ }^{2}$}

ECS induced paraplegia (seven casualties) necessitated the random elimination of normal $\mathrm{Ss}$ from the appropriate groups to equalize $\mathrm{N}$ across groups for statistical analyses. The data presented are from the 10 remaining $\mathrm{Ss}$ in each group. Figure 1 shows the percent correct performance in blocks of four trials for each group. The Ss in Groups SEI and SEO quickly learned to avoid the treatment box and continued to respond to the treatment free box throughout testing at a much higher level than the other groups. The curves of Groups EI and EO show only a gradual rise in performance. Group $S$ fell between these two extremes. In terms of total percent avoidance responses, the 


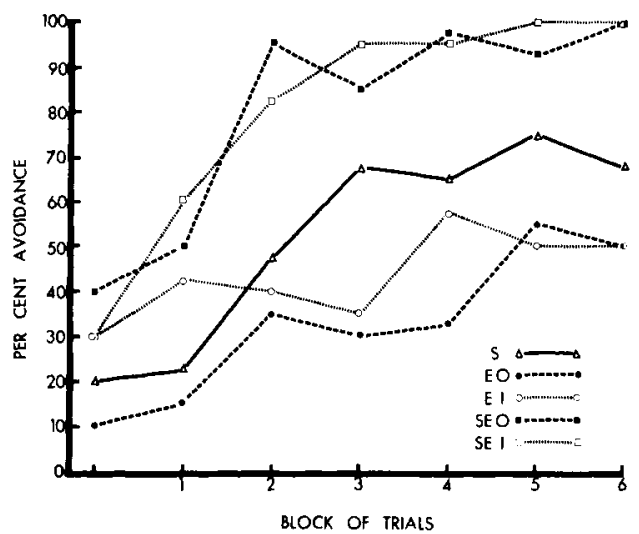

Fig. 1. Percentage of choices of non-treatment box for each group in four trial blocks. The first data points are the percent responses, on the first trial of the last training day, to the goal box subsequently defined as "correct."

groups from highest to lowest were ordered: SEI, SEO, $\mathrm{S}, \mathrm{EI}$, and EO, and the percentages were: $89,87,58,46$, and 36 respectively. An analysis of the arcsin of total percent avoidance responses of each $S$ revealed highly significant differences among groups: $F=7.35, p<.001$, $\mathrm{df}=4,49$. The Duncan (1955) range test yielded significant differences between the comparisons of Groups SEI and SEO with all other groups but not between each other (i.e., SEI vs. $S, p<.05$; SEI vs. EI, $p<.01$; SEI vs。 EO, $p<.001$; SEO vs. S, $p<.05$; SEO vs. EI, $p<.01$; SEO vs. EO, $p<.001)$. The other comparisons, among Groups S, EI, and EO failed to reach significance.

The fact that Groups SEI and SEO had significantly more responses to the treatment free box than did any of the other groups indicates that ECS following grid shock in the goal box had a facilitative effect on avoidance learning. This result is incompatable with the ECS retrograde amnesia hypothesis. In this respect the results are in agreement with those of Coons \& Miller (1960) although they used latency rather than choice as the response measure.

That Groups SEI and SEO had significantly higher avoidance levels than Group $S$ indicates that the aversive components of the treatment arm could not have been due to grid shock alone. Conversely the relative inferiority of Groups EI and EO in avoiding the treatment arm would indicate that the behavior in Groups SEI and SEO cannot be explained by ECS avoidance either. These findings argue against a simple ECS fear or a simple competing response hypothesis, i.e., that Groups EI and EO performed at such a consistently low level of ECS avoidance suggests that ECS itself is not a strong aversive stimulus (cf. McGaugh \& Madsen, 1964) or that any fear responses it may produce do not readily become conditioned to innocuous conditions that precede it, or greatly interfere with responses that result in it. The lack of significant differences due to place of ECS administration does not agree with the results of Adams \& Lewis $(1962 \mathrm{a}, \mathrm{b})$ although situational differences and the ceiling effect of Groups SEI and SEO preclude comment on this discrepancy.

However the results do suggest some sort of conditioning process, whereby the grid shock is the CS, and ECS has the properties of an aversive UCS. This notion fits reasonably well with the high performance of the grid shock-ECS groups, assuming that the aversive factors of ECSare prominent only where a salient and/or "similar" CS is used. This study is, then, yet another indication that repeated ECS trials (within the response-ECS interval used) will not suffice as a test of the trace consolidation hypothesis of memory.

\section{References}

Adams, H. E., \& Lewis, D. J. Electroconvulsive shock, retrograde amnesia, and competing responses. J. comp. physiol. Psychol. $1962 a, 55,299-301$

Adams, H. E., \& Lewis, D. J. Retrograde amnesia and competing responses. J. comp, physiol. Psychol., 1962b, 55, 302-305.

Coons, E. E., \& Miller, N. E. Conflict versus consolidation of memory traces to explain "retrograde amnesia" produced by ECS. J. comp. physiol. Psychol., 1960, 53, 524-531.

Duncan, D. B. Multiple range and multiple $F$ test. Biometrics, $1955,11,1-42$.

McGaugh, J. L., \& Madsen, M. C. Amnesic and punishing effects of electroconvulsive shock. Science, 1964, 144, 182-183.

\section{Notes}

1. Data herein were reported at the Midwestern Psychological Association meeting, St. Louis, 1964.

2. A pilot study duplicated the results of Groups SEO, EO and S. 